\title{
CEPSTRAL ANALYSIS IN TOOL MONITORING
}

\author{
Igor Vilcek, Jan Madl \\ Czech Technical University in Prague, Faculty of Mechanical Engineer- \\ ing, Dept. of Manufacturing Technology, Technická 4, 16607 Prague 6 \\ , CZECH REPUBLIC \\ phone: +420224352611 \\ e-mail:vilcek@fsid.cvut,cz,madl@fsid.cvut.cz.
}

Utilization of force signals to achieve on line drill wear monitoring is presented in this paper. After consulting the available literature it is obvious, that only some features of force component are proposed for drill wear monitoring .The really important task in drilling operations is to avoid catastrophic failure. It is desirable to make the most economic use the cutting tool without reaching catastrophic failure. Traditionally, the usual approach to tool monitoring the drilling was to the detect breakage as fast as possible and avoid overloads in the machine tool. These strategies are not enough to ensure the optimum economic performance of the machining process.

Therefore, the primary objective of this lecture assesses the feasibility of using force signature analysis as means for monitoring tool wear.

Keywords: cepstral analysis, monitoring, machining, cutting forces, harmonics

\section{INTRODUCTION}

Requirements for flexible manufacturing have been increasing in the last years. In order to insure effective operation of expansive manufacturing equipment, which has to run automatically and unattended, tool monitoring is important. Therefore, the essential problem to be overcome to achieve the full potential of unmanned machining is the development of effective and reliably sensors systems to monitoring the process and corrective action in case abnormal operation. With increasing wear in the twist drill margin wear causes the increase of the frictional forces between the margin and machining hole wall and leads to tensional vibrations in the cutting tool. This in turn will cause further tool wear and vibrations. If the cyclic process continuous catastrophic failure will occur at a short time. At the moment when these tensional vibrations appear, it is the appropriate time for drill bit change, since from this point on, wear increases rapidly due to the phenomenon of tensional vibrations.

Quante et al [4] recognized the importance of sensing vibrations in the twist drill for wear monitoring as a mean to overcome the difficulties of the slight sensitivity of the static component of the thrust force to wear. They proposed the use of 
the distance sensors without contact measuring deflection of the drill in a plane normal to the drill axis. A synchronization device was attached to the spindle emitting 256 pulses per revolution. The signal of the distance sensor was hight pass filtered at $60 \mathrm{~Hz}$ to avoid the effect of the spindle speed frequency at $12 \mathrm{~Hz}$. An increase ranging to 5 to 8 times in the signal for a worn drill with respect to the initial value when sharp was reported. The advantage of the system is that since it senses without contact at the tool shank, as was the case with eddy current torque sensors proposed Brinksmeier et al [5], it can by applied to almost any existing machine tool without structural changes. The sensors are expensive and do not interfere with machining process.

The proposed approaches of cutting force signature analysis to be investigated and compared are the following:

- Static component of cutting forces signal

One of the problems observed in the literature on the use of signal features from the static component is the occurrence of false alarms due to the stochastic character of the cutting process and especially due to variations in hardness along the workpiece. Subramian and Cook, 1977 et al. [13] established 5 percent as the maximum allowable variation in workpiece hardness for the static component of torque and thrust force to be used successfully as variables for drill wear sensing. To overcome this limitation and solve the possible false alarm problem, the thrust force-torque ratio is proposed as a method for detected the wear by means of the DC component of the signal.

- Dynamic component of cutting forces signal: Frequency analysis Analysis of the dynamic component of the cutting force signal has been neglected hitherto in most approaches to tool condition monitoring in drilling. This method is expected to be sensitive for detecting tool wear. Frequency domain methods will be applied and several analysis techniques will be explored such as the power spectrum, the power cepstrum, cross spectrum.

- Tool failure prediction

It has been observed that violent and sudden oscillations occur in force signal when the tool is reaching the end of its life and is about to fail. This phenomenon is thought to be produced by a certain wear mechanism occurring at the end of tool life, when severe wear is already present, and leading invariably to catastrophic failure. To detect this phenomenon and thus predict tool failure the derivative of the cutting force signal are thought to be sensitive. Other ways of detecting the wear mechanism leading to failure will be explored; by means of frequency domain methods such as cepstral analysis and coherence function among both thrust force and torque signals.

\section{SOFTWARE USED FOR EXPERIMENTS}

Matlab software with a Real Time Toolbox was used for data acquisition in order to sample the cutting force signals from the dynamometer. The sampled data was saved on the hard disk of the computer for further processing and analysis. For this analysis of sampled data the Editace program, developed at the Department of Mechanical 
Technology of the Czech Technical University in Prague, was used. This program is based on Matlab and is able to import the sampled data.

The measured signal can be displayed and edited, unwanted parts of the signal can be deleted, and zero can be adjusted. The basic statistical parameters of the selected part of the signal can be computed regression analysis can be performed and a curve fitted (Novak and Madl 2000) [2]. with.

The advantage of this program is that it is very user friendly and easy to work

This program is used to determine empirical data for dynamic measurements of the forces and torques. This data can be utilised for optimisation of the machining process.

The results of the program should be:

- A graph of measured values

- A graph of the gradient of measured values

- A file of statistical parameter(s)

- Values of dependent parameters in the selected regression model

This software is used for drilling, milling and turning.

\section{EXPERIMENTAL DEVICE}

A series of drilling and milling experiments were carried out.

Cutting conditions :

HSS twist drill of diameter 2,2 and $4,5 \mathrm{~mm}$.

Work material - ISO 683/XIII-86 and $11 \mathrm{Cr} 16 \mathrm{Ni2}$ Mo1 Feed: 0,1mm per rev.

Number of revolutions: 2500 per min.

Dry drilling.

The thrust force and torque produced by the drilling process were measured by means of a type 9272 Kistler four-component piezoelectric dynamometer.

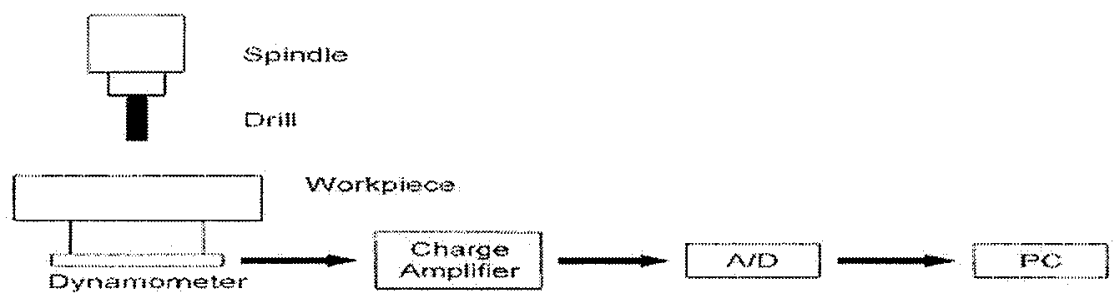

Figure 1

The signals were amplified by Vibrometer A.G type TA-3/C charge amplifiers with two individual channels. The amplified signals were sampled using a data acquisition $\mathrm{c}$ ard on the hard disk of the $\mathrm{c}$ omputer for further a nalysis. A s chematic diagram of the experimental set-up is shown Figure1. For data acquisition Matlab software with a Real Time Toolbox are used in order to sample the cutting force signals from the dynamometer. The sampled data was saved on the hard disk of the 
computer for further processing and analysis. The Editace program was used to analysis the sampled data.

\section{FAILURE PREDICTION BY MEANS OF CEPSTRAL ANALYSIS}

The harmonics of the basic rotational frequency in fact dominates the spectrum when instability prior to failure is reached. The stronger the unstable wear phenomenon the larger the effect of harmonics.

Therefore, an efective strategy for twist drill failure prediction would be to detect the occurrence of the harmonics and to determine their presence by cepstral analysis.

Power spectrum density estimate of the thrust force for the first hole (left) and for the last hole (right) is shown in Fig. 2

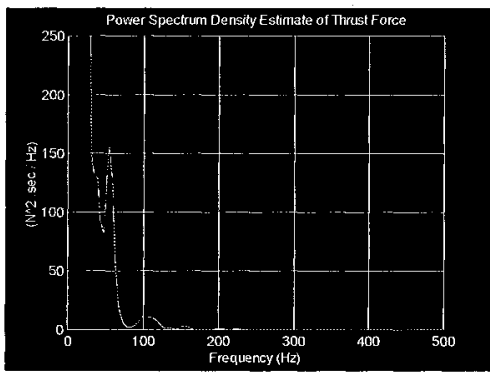

Figure 2

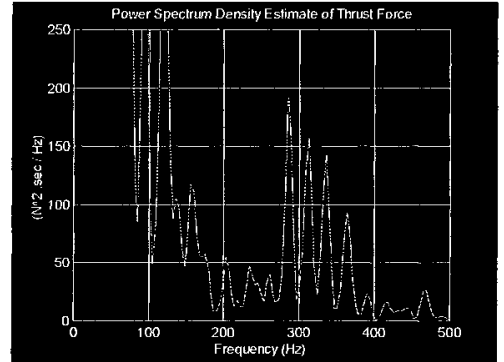

Figure 3

The cepstrum, strictly speaking the power cepstrum, is defined as the power spectrum of the logarithm of the power spectrum (Randall 1977) [1]. If the forward Fourier transform of a time function $\mathrm{fx}(\mathrm{t})$ is denoted by:

$$
F x(t)=F\{f x(t)\}
$$

then the power spectrum may be represented by

$$
F x \boldsymbol{x}(f)=|F x(f)|^{2}
$$

And the cepstrum

$$
\boldsymbol{C x}(\tau)=|\boldsymbol{F}\{\log [\boldsymbol{F x} x(f)]\}|^{2}
$$

The independent variable $\tau$ of the cepstrum is called the Quefrency, though it has the dimensions of time. The characteristic feature of the cepstrum is its ability to detect and give a measure of the phenomena, which exhibit periodicity in the spectrum, such as the harmonics. In particular, in complex signals containing a mixture of different families of harmonics, separation of the various periodicities is greatly facilitated by performing the second Fourier transform to obtain the cepstrum. A point about which different opinions exist is whether the second Fourier transform should be a forward or an inverse transform. However this is not important, since the result is identical except for a scaling factor. If an inverse Fourier transform is used, for the sake of consistency, the cepstrum is represented by 


$$
C x(\tau)=\left|F^{-1}\{\log [F x x(f)]\}\right|^{2}
$$

\section{FAILURE PREDICTION BY MEANS OF THE COHER- ENCE FUCTION BETWEEN THRUST FORCE AND TORQUE SIGNALS}

An approach is put forward for catastrophic failure prediction, based on the detection of the above mentioned specific wear mechanism which occurs in the third stage of tool life, the stage of final accelerated wear (catastrophic failure).

The coherence function indicates the extent to which two signals are correlated with each other. In other words, it can be said that the coherence function gives a measure of the validity of the assumption that both signals result from the same particular generating mechanism or source.

The coherence function $\gamma_{x y}(f)$ is defined by:

$$
\gamma_{x y}{ }^{2}(f)=\frac{\left|F_{x y}(f)\right|^{2}}{F_{x x}(f) \cdot F_{y y}(f)} ; \quad 0 \leq \gamma_{x y}(f) \leq 1
$$

where $F_{x y}(f)$ and $F_{y y}(f)$ are the power spectra of each signal, also often referred to as auto spectra where $F_{x y}(f)$ is the cross spectrum. The cross spectrum $F_{y y}(f)$ of $f_{x}(t)$ and $f_{y}(t)$ is the forward Fourier transform of the cross correlation function $R_{x y}(\tau)$, which is, in turn, defined by the equation:

$$
R_{x y}(\tau)=\lim _{T \rightarrow \infty} \frac{1}{T} \int_{-T / 2}^{-T / 2} f_{x}(t) \cdot f_{y}(t+\tau) . d t
$$

This gives a measure of the extent to which $t$ wo signals correlate with each other as a function of the time displacement between them. The cross spectrum can alternatively be obtained from the individual Fourier spectra $F_{x}(f)$ and $F_{y(f)}$ as follows:

$$
F_{x y}(f)=F_{x}^{*}(f) \cdot F_{y}(f)
$$

where $F_{x}{ }^{*}(f)$ is the complex conjugate of $F_{x}(f)$.

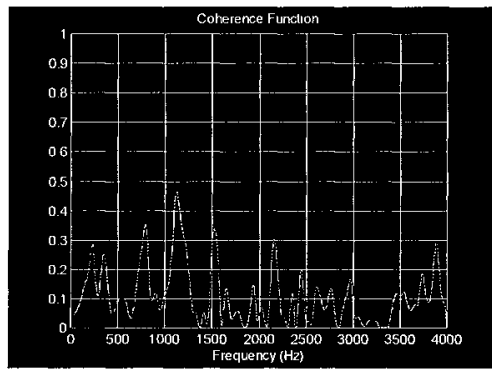

Figure 4

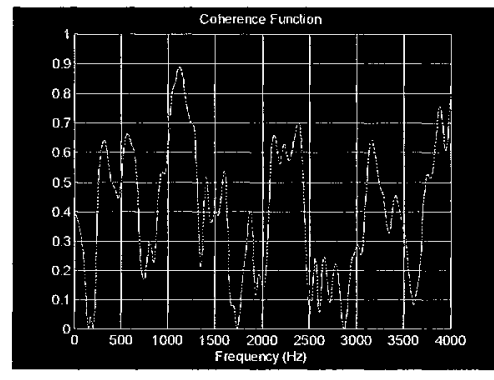

Figure 5 
Therefore, three cases are possible. The coherence function can be zero, one or greater than zero and less than unity. If $g_{x y}{ }^{2}(f)=0$ for all frequencies the two signals are completely uncorrelated. In case $g_{x y}{ }^{2}(f)=1$ for all frequencies the two signals are completely correlated. If $\mathrm{g}_{\mathrm{xy}}{ }^{2}(\mathrm{f})$ is between zero and one for all frequencies, one or more of the following conditions exist: a) even when the two signals $y(t)$ and $x(t)$ are caused partially by the same phenomenon or generating source, each is also caused in part by the other phenomena which affect it individually but do not affect the other signal, b) extraneous noise is present in the measurements, c) bias errors are a spectral estimation.

The coherence function for the first hole is shown Figure 4 or the last hole is shown in Figure 5

\section{CONCLUSION}

Some methods are presented for predicting of catastrophic failures in drilling, based on the detection of a specific wear mechanism operating at the end of tool life when severe wear is present and leading unavoidably to catastrophic failure. The basic characteristic of this wear mechanism is that it excites strongly tensional vibrations of the cutting tool. The proposed method relies in detecting the rise of harmonics in the spectrum of the torque signal when the wear mechanism begins to operate by means of the cepstral analysis (coherence function, power spectrum density estimate of the thrust force and torque)

\section{REFERENCES}

1. Randall, R.B., Application of B \& K Equipment to Frequency Analysis, Bruiel and Kjaer information, Denmark, 1977

2. Novak, P. \& Madl, J, Effective Evaluation of Measured Dynamic Values of Cutting Forces and Torques, Manufacturing Technology, 2001, ISSN 112386

3. El -Wardany, T.I. \& Gao, D. \& Elbestawi, M.A, Tool Condition Monitoring in Drilling Using vibration Signature Analysis, International Journal of Machine Tools and Manufacture, Vol. 36 No. 6, 1996, pp. 687-711

4. Quante, F. \& Fehrnbach, H. \& Meir, H.,-E., Automatische Uberwachung Rotierender Werkzeuge mit Abstands- und Schwingungssensoren in der Spanabhebenden Fertigung, Technisches Messen, 50. Jahrgang Heft 10, 1983, pp. 367-371

5. Brinksmeier, E., Prediction of Tool Fracture in Drilling, Annals of the CIPR, Vol.39, No. 1, 1990, pp 97-100

6. Cook, N.H., Tool Wear Sensors, Wear, Vol. 62, 1980, pp. 49-57

7. Bendat, Julius S. \& Piersol, Allan G., Random data: Analysis and measurement procedures, New York, Wiley-Interscience, 1971

8. Boston O.W. and Gilbert, W.W., The Torque and Thrust of Small Drills Operating in Varius Metals, Transaction of the ASME, 1936, pp 79-89

9. Armarego, E.JA. \& Brown, R.H., The Machining of Metals, Prentice-Hall, New Jersey, 1969

10. Merritt, H.E., Theory of Self-Excited Machine Tool Chatter, Transactions of the ASME, Series B, Journal of Engineering for Industry, Vol. 87, 1965, p. 447

11. Braun, S. \& Lenz, E. \& Wu, C.L., Signature Analysis Applied to Drilling, Transactions of the ASME, Journal of Engineering for Industry, Apr., 1982, pp. 268-276

12. Conrad, Charles J. \& McClamroch, N. Harris, The Drilling Problem: A Stochastic Modeling and Control Example in Manufacturing, IEEE Transactions on Automatic Control, Vol. AC-32, No. 11, 1987, pp. $947-958$

13. Subramanian, K. \& Cook, N.H., Sensing of drill Wear and Prediction of Tool Life, Transactions of the ASME, Journal of Engineering for Industry, May. 1977, pp. 295-301 